\title{
An efficient implementation of iterative adaptive approach for source localization
}

\author{
Gang $\mathrm{Li}^{1{ }^{*}}$, Hao Zhang ${ }^{1}$, Xiqin Wang ${ }^{1}$ and Xiang-Gen Xia ${ }^{2}$
}

\begin{abstract}
The iterative adaptive approach (IAA) can achieve accurate source localization with single snapshot, and therefore it has attracted significant interest in various applications. In the original IAA, the optimal filter is performed for every scanning angle grid in each iteration, which may cause the slow convergence and disturb the spatial estimates on the impinging angles of sources. In this article, we propose an efficient implementation of IAA (EIAA) by modifying the use of the optimal filtering, i.e., in each iteration of EIAA, the optimal filter is only utilized to estimate the spatial components likely corresponding to the impinging angles of sources, and other spatial components corresponding to the noise are updated by the simple correlation of the basis matrix with the residue. Simulation results show that, in comparison with IAA, EIAA has significant higher computational efficiency and comparable accuracy of source angle and power estimation.
\end{abstract}

Keywords: sparse recovery; iterative adaptive approach; source localization.

\section{Introduction}

Source localization is a fundamental problem in a wide range of applications including communications, radar, and acoustics, and many algorithms have been presented in the literature during recent decades. The Fourier-based algorithms suffer from the low resolution and the high sidelobes. Some methods based on subspace processing, e.g., Capon beamforming [1], MUSIC [2], ESPRIT [3], and other subspace-based algorithms [4,5], provide superresolution for uncorrelated sources with sufficient number of snapshots. However, in the case of few snapshots, the performances of these subspace-based methods will degrade sharply.

Recently, the source localization problem has been converted into a sparse recovery framework, because the number of actual sources of interest is generally much smaller than the number of potential source locations in the region to be observed. A kind of algorithms of sparse recovery is based on iterative weighted least squares, e.g., the FOCal Underdetermined System Solver (FOCUSS)

*Correspondence: gangli@tsinghua.edu.cn

${ }^{1}$ Tsinghua National Laboratory for Information Science and Technology

(TNList), Department of Electronic Engineering, Tsinghua University, Beijing

100084, China

Full list of author information is available at the end of the article
[6], the Sparse Learning via Iterative Minimization (SLIM) [7], the iterative adaptive approach (IAA) [8], etc. Here, we are interested in IAA, which is able to provide accurate source localization with single snapshot and has attracted significant interest in various applications [9-11]. IAA is non-parametric and it achieves accurate estimates of angles and powers of the sources by iterative operations [8]. The spatial component on every potential angle is estimated by optimal filtering, which passes the signal from the current angle without distortion and fully suppresses the interferences from other angles. The iteration is terminated when the norm of the difference between two successive spatial estimates is smaller than a certain threshold. However, it is time consuming to perform optimal filtering on all potential angles, since in general we are only interested in several angles where the actual sources are located. Moreover, the excessive estimation of the spatial components on the angles that are outside the actual source position set may result in a slow convergence. In this article, we propose an efficient implementation of IAA (EIAA) by modifying the use of the optimal filtering, i.e., in each iteration, the optimal filter is only utilized to estimate the spatial components likely corresponding to the actual signal sources, and other spatial components corresponding to the noise are updated by the simple correlation of the basis matrix with the residue. It will be shown that

\section{实}


EIAA has significant faster convergence speed and comparable accuracy of source angle and power estimation. In $[12,13]$, two fast implementations of IAA have been proposed by using the matrix computation technique such as Gohberg-Semencul decomposition, etc. It is noted that the way of the computational burden reduction in this article is different from $[12,13]$ : herein, we focus on reducing the number of running optimal filtering procedures, while [12, 13] focus on improving the computational efficiency of the optimal filtering procedure. In addition, similar to the algorithms mentioned above, we are only interested in the unambiguous angle solution, which depends on the ratio of interelement spacing of the array to the wavelength. In the case that the angle ambiguity occurs, we refer to [1416] for resolving the ambiguity.

The remainder of this article is organized as follows. The signal model and the original IAA are introduced in Section 2. The EIAA algorithm is proposed in Section 3. The proposed EIAA is evaluated by some simulations in Section 4. Concluding remarks are presented in Section 5.

\section{Signal model and IAA}

Suppose that $K$ potential far-field narrowband signals are impinging on an $M$-element array from directions $\left\{\theta_{1}, \theta_{2}, \ldots, \theta_{K}\right\}$. In single snapshot case, the output measurement vector of the array can be expressed as

$$
\mathbf{y}=\mathbf{A s}+\mathbf{e}
$$

where $\mathbf{A}$ is the $M \times K$ basis matrix and is defined by $\mathbf{A}=$ $\left[\mathbf{a}\left(\theta_{1}\right), \mathbf{a}\left(\theta_{2}\right), \ldots, \mathbf{a}\left(\theta_{K}\right)\right], \mathbf{s}$ is the $K \times 1$ vector denoting the complex amplitudes of the sources, $\mathbf{e}$ is the additive noise. Considering an $M$-element linear array as shown in Figure 1, the $k$ th column of $\mathbf{A}$ corresponding to the potential source direction $\theta_{k}$ can be represented by

$$
\begin{aligned}
\mathbf{a}\left(\theta_{k}\right)= & {\left[e^{-j 2 \pi x_{1} \cos \left(\theta_{k}\right) / \lambda}, e^{-j 2 \pi x_{2} \cos \left(\theta_{k}\right) / \lambda},\right.} \\
& \left.\ldots, e^{-j 2 \pi x_{M} \cos \left(\theta_{k}\right) / \lambda}\right]^{T},
\end{aligned}
$$

where $\left\{x_{1}, x_{2}, \ldots, x_{M}\right\}$ are the positions of the $M$ elements of the array, respectively, $\lambda$ is the wavelength, $(\cdot)^{T}$ denotes transpose. In sparse recovery framework, the potential

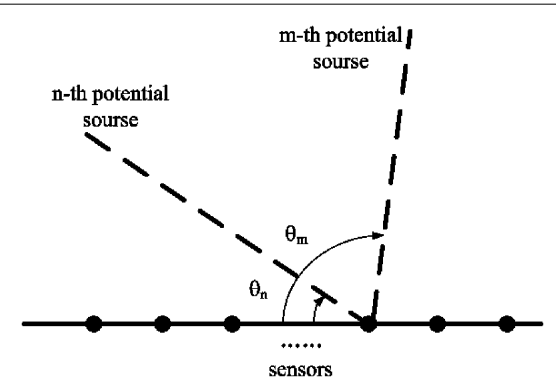

Figure 1 The geometry of sensors and sources.

source number $K$ is rather considered to be the number of discretized angle grids. Assume that $\mathbf{s}$ is sparse, i.e., the number of actual sources is much smaller than $K$. Consider the line-spectrum model and let $\mathbf{P}=\operatorname{diag}\left\{p_{1}, p_{2}, \ldots, p_{K}\right\}$, whose $k$ th diagonal element $p_{k}$ contains the power at $k$ th scanning angle grid. The problem of interest is recovering the spatial components $\left\{p_{1}, p_{2}, \ldots, p_{K}\right\}$, and the positions and the amplitudes of the peaks of $\left\{p_{1}, p_{2}, \ldots, p_{K}\right\}$ directly provide the locations and the powers of the sources. IAA [8] achieves this goal as summarized in Table 1 , where the superscript $(i)$ denotes the $i$ th iteration.

\section{Efficient implementation of IAA}

It is noted that step (b) in Table 1 gives an optimal filter in terms of $\theta_{k}$, which reserves the signal from angle $\theta_{k}$ without distortion and fully suppresses the interferences (signals from other angles). In each iteration the optimal filtering is performed $K$ times for all angles $\left\{\theta_{1}, \theta_{2}, \ldots, \theta_{K}\right\}$. This is computationally extravagant, because in general we are only interested in the angle set where the actual sources are located. Moreover, for the index $k$ corresponding to $\theta_{k}$ outside the angle set of actual sources, $p_{k}$ most likely depends on the noise power, and the iterative estimation of $p_{k}$ may increase the actual number of iterations required for the convergence. Based on the above observations, we modify IAA as described in Table 2.

The main difference between the proposed EIAA and the original IAA lies in the estimation of spatial components that are outside the actual source location set. As seen from step (b) in Table 2, $\left\{\theta_{k}\right.$ with index $\left.k \in \Lambda^{(i)}\right\}$ are considered to be likely angle candidates where actual

Table 1 IAA algorithm.

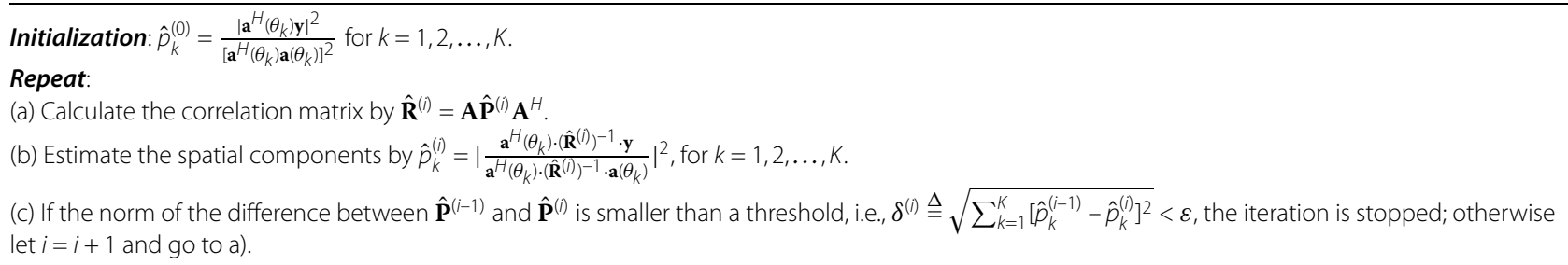


Table 2 EIAA algorithm.

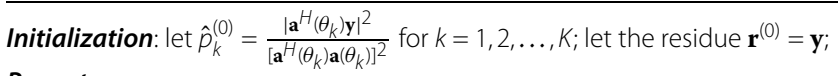

Repeat

(a) Calculate the correlation matrix by $\hat{\mathbf{R}}^{(i)}=\mathbf{A} \hat{\mathbf{P}}^{(i)} \mathbf{A}^{H}$;

Let the index support set $\Lambda^{(i)}=\varnothing$ and the principal spatial component set $\Gamma^{(i)}=\varnothing$.

(b) While the relative residue is larger than a threshold, i.e., $\frac{\left\|\mathbf{r}^{(i)}\right\|_{2}^{2}}{\|\mathbf{y}\|_{2}^{2}}>\xi$

Find the index $n_{1}$ corresponding to the largest entry in the vector $\left[\hat{p}_{1}^{(i)}, \hat{p}_{2}^{(i)}, \ldots, \hat{p}_{k}^{(i)}\right]$;

Expand the index support set by $\Lambda^{(i)}=\left\{\Lambda^{(i)}, n_{1}\right\}$;

Expand the principal spatial component set by $\Gamma^{(i)}=\left\{\Gamma^{(i)},\left|\frac{\mathbf{a}^{H}\left(\theta_{n,}\right) \cdot\left(\hat{\mathbf{R}}^{(i)}\right)^{-1} \cdot \mathbf{y}}{\mathbf{a}^{H}\left(\theta_{n \ell}\right) \cdot\left(\hat{\mathbf{R}}^{(i)}\right)^{-1} \cdot \mathbf{a}\left(\theta_{n l}\right)}\right|^{2}\right\}$;

Calculate the residue by $\mathbf{r}^{(i)}=\mathbf{y}-\left(\mathbf{A}_{\Lambda^{(i)}}^{H} \mathbf{A}_{\Lambda^{(i)}}\right)^{-1} \mathbf{A}_{\Lambda^{(i)}}^{H} \mathbf{y}$, where the matrix $\mathbf{A}_{\Lambda^{(i)}}$ consists of the columns of $\mathbf{A}$ with indices $k \in \Lambda^{(i)}$;

Update the spatial estimate by $\hat{p}_{k}^{(i)}=\frac{\left|\mathbf{a}^{H}\left(\theta_{k}\right) \mathbf{r}^{(i)}\right|^{2}}{\left[\mathbf{a}^{H}\left(\theta_{k}\right) \mathbf{a}\left(\theta_{k}\right)\right]^{2}}$, for $k=1,2, \ldots, K$.

end While

(c) Restore the principal spatial components by $\hat{p}_{k}^{(i)}=\Gamma^{(i)}(k)$, for $k \in \Lambda^{(i)}$.

(d) If the norm of the difference between $\hat{\mathbf{P}}^{(i-1)}$ and $\hat{\mathbf{P}}^{(i)}$ is smaller than a threshold, i.e., $\delta^{(i)} \triangleq \sqrt{\sum_{k=1}^{K}\left[\hat{p}_{k}^{(i-1)}-\hat{p}_{k}^{(i)}\right]^{2}}<\varepsilon$, the iteration is stopped; otherwise let $i=i+1$ and go to a).

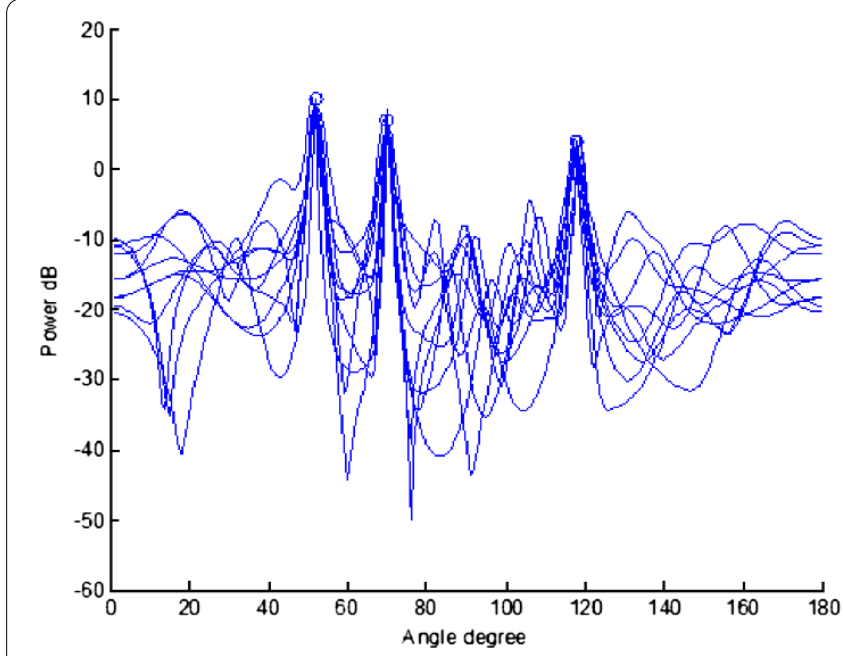

(a)

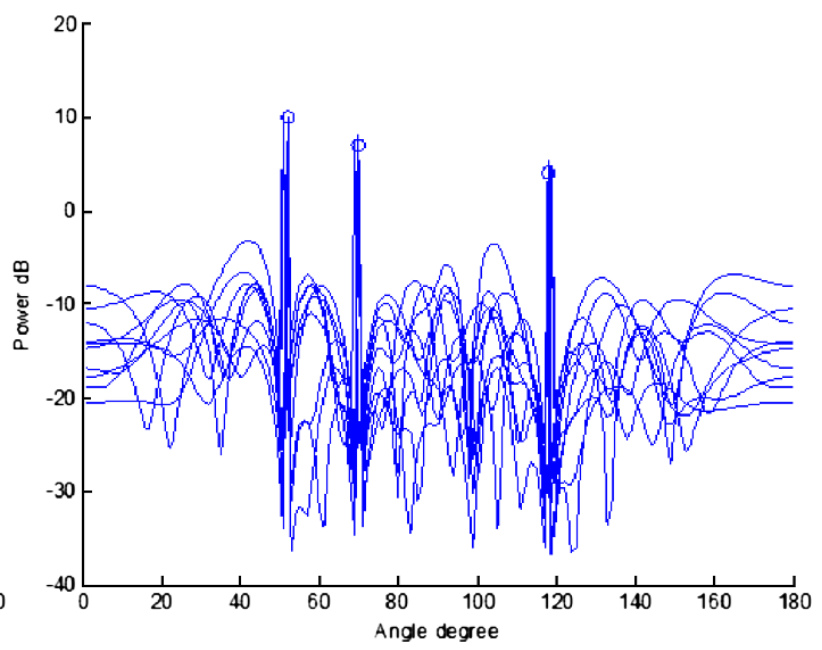

(b)

Figure 2 Spatial estimation results by 10 Monte-Carlo trials. (a) IAA estimates, (b) EIAA estimates

Table 3 Performances of IAA and EIAA for various SNR.

\begin{tabular}{|c|c|c|c|c|c|}
\hline$\overline{\text { SNR }}$ & $0 \mathrm{~dB}$ & $5 \mathrm{~dB}$ & $10 \mathrm{~dB}$ & $15 \mathrm{~dB}$ & $20 \mathrm{~dB}$ \\
\hline $\mathrm{er}_{\text {EIAA }}^{\text {angle }}-\mathrm{er}_{\text {IAA }}^{\text {angle }}$ & $-0.297^{\circ}$ & $-0.114^{\circ}$ & $-0.107^{\circ}$ & $-0.015^{\circ}$ & $-0.004^{\circ}$ \\
\hline $\mathrm{er}_{\text {EIAA }}^{\text {power }}-\mathrm{er}_{\text {IAA }}^{\text {power }}$ & -0.087 & -0.393 & -0.543 & -0.489 & -0.373 \\
\hline RTR of EIAA and IAA & 0.131 & 0.118 & 0.081 & 0.072 & 0.070 \\
\hline
\end{tabular}

sources are located. Then, the spatial components corresponding to the actual source locations are updated by optimal filtering, and other spatial components corresponding to the noise are updated by simple correlation of the columns of basis matrix with the residue. This implies that the excessive estimation of noise components is avoided. Compared with the original IAA, EIAA can significantly reduce the computational burden thanks to the following facts: (1) In each iteration, the required times of optimal filtering procedure is equal to the number of the selected principle components in step (b) of Table 2. The step (b) of Table 2 is finished by the residual energy threshold, for example, in practice it is reasonable to let $\xi=0.05$, which implies that the relative residue energy is smaller than 5\%. In high SNR case, it is believable that the number of the selected principle components in step (b) of Table 2 is equal to the number of the actual sources; for lower SNR, the number of the selected principle components in step (b) of Table 2 may be slight larger than the number of the actual sources because the signal-subspace and the noise-subspace become undistinguishable. Any- 


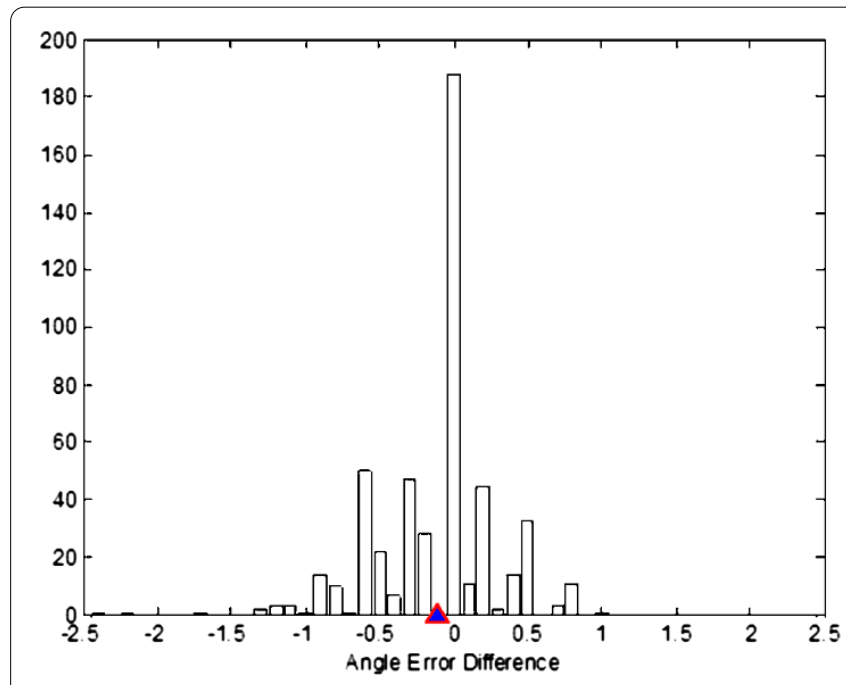

(a)

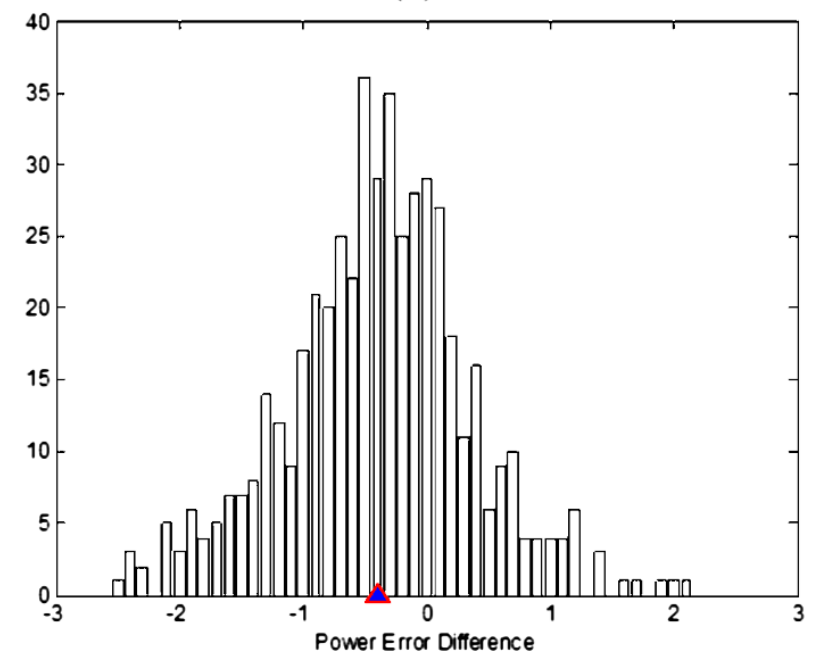

(b)

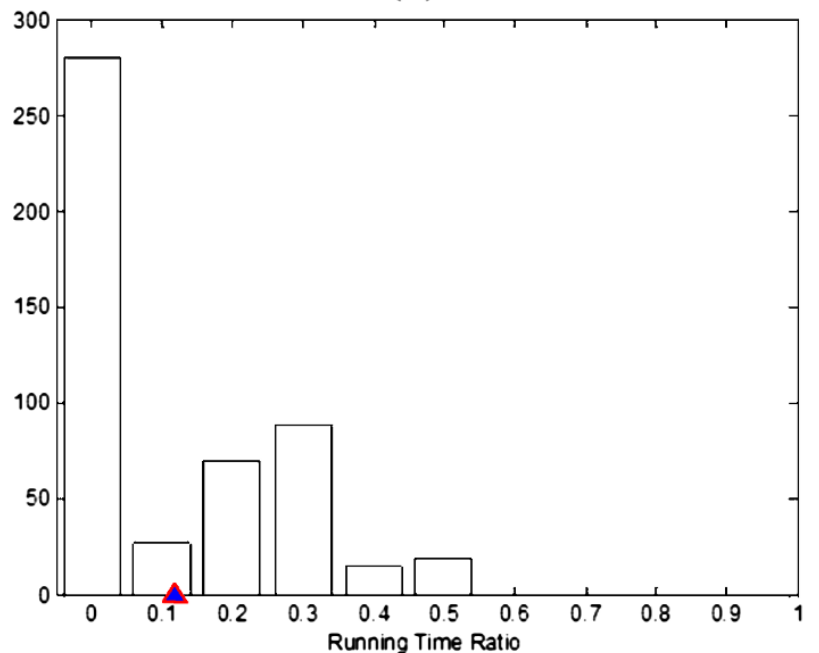

(c)

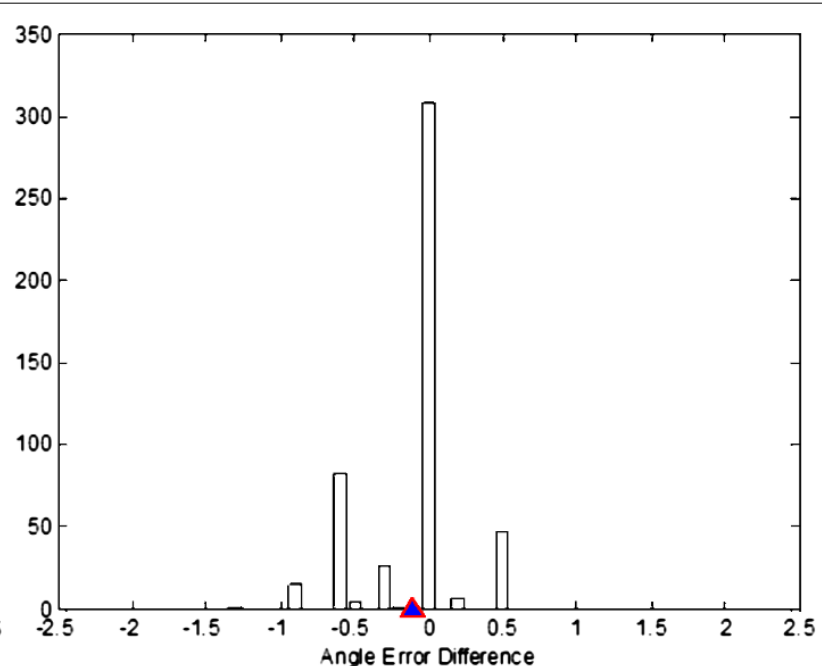

(d)

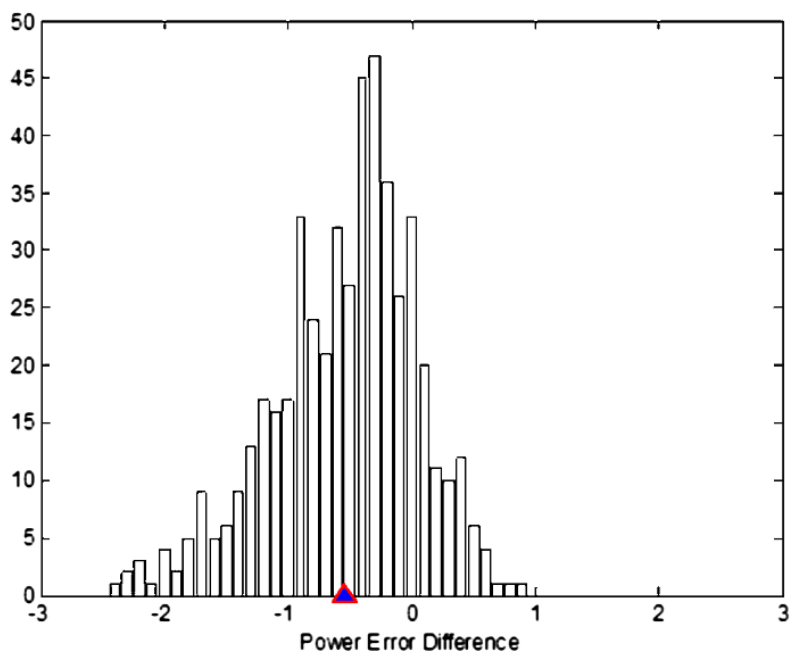

(e)

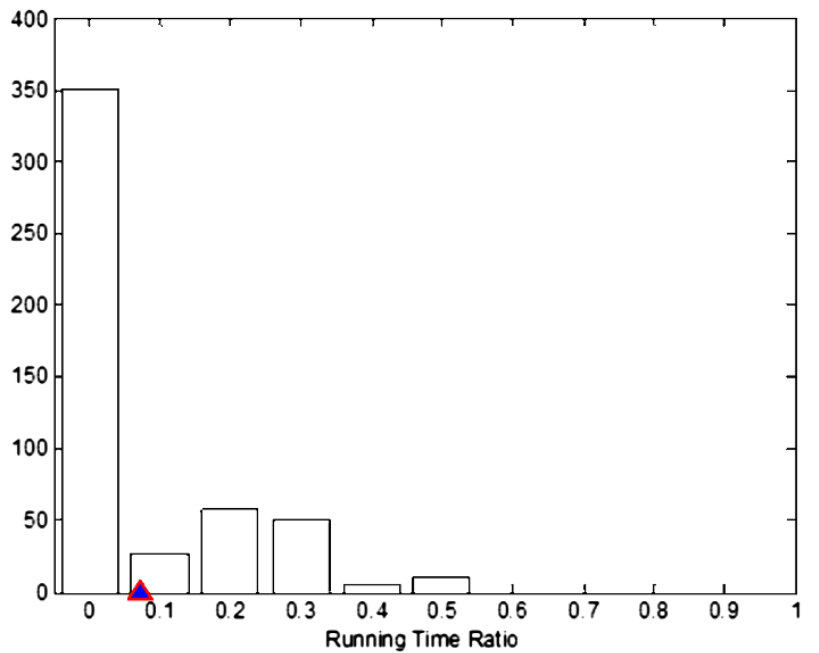

(f)

Figure 3 Performance comparison of IAA and EIAA. (a-c) For SNR $=5 \mathrm{~dB}$; (d-f) for SNR $=10 \mathrm{~dB}$. (a, d) Angle error difference er $\mathrm{er}_{\mathrm{EIAA}}^{\text {angle }}-\mathrm{er}_{\mathrm{IAA}}^{\text {angle }}$; $(\mathbf{b}, \mathbf{e})$ power error difference er $e_{\text {EIAA }}^{\text {power }}-\operatorname{er}_{\text {IAA }}^{\text {power }} ;(\mathbf{c}, \mathbf{f})$ RTR of EIAA and IAA. 
way, the number of the selected principle components, i.e., the required times of optimal filtering procedure, is usually much smaller than $K$, which is guaranteed by the prior assumption of sparse signal property. (2) The relaxation of the estimation of spatial components corresponding to noise leads to stable and fast convergence.

\section{Simulations}

In this section, some examples are provided to evaluate the performance of the proposed EIAA in single snapshot case. Consider a uniform linear array of $M=14$ sensors with the interelement spacing $\lambda / 2$. The additional noise is assumed Gaussian with zero mean and variance $\sigma^{2}$, and the SNR is defined as $10 \log _{10}\left(\sum_{n=1}^{L} p_{n} /\left(L \sigma^{2}\right)\right)$. The angle scanning grid is uniform in the range from $1^{\circ}$ to $180^{\circ}$ with $1^{\circ}$ increment between adjacent angle candidates.

Assume that there are three sources at $52^{\circ}, 70^{\circ}$, and $118^{\circ}$ with $p_{1}=10 \mathrm{~dB}, p_{2}=7 \mathrm{~dB}$, and $p_{3}=4 \mathrm{~dB}$ powers, respectively. For $\mathrm{SNR}=10 \mathrm{~dB}$, Figure 2 shows the spatial estimates of IAA and EIAA. The true source locations are indicated by circles, and the results of ten Monte-Carlo trials are plotted. One can see that both of IAA and EIAA can accurately indicate the locations and powers of the sources, while EIAA shows sharper peaks.

The performances of IAA and EIAA are compared via histograms over 1000 trials in Figure 3, where $\mathrm{SNR}=5 \mathrm{~dB}$ for $(\mathrm{a}-\mathrm{c})$ and $\mathrm{SNR}=10 \mathrm{~dB}$ for $(\mathrm{d}-\mathrm{f})$. The thresholds in Table 2 are set by $\varepsilon=0.01$ and $\xi=0.05$. The source localization error is defined by er ${ }^{\text {angle }} \triangleq \sqrt{\sum_{i=1}^{3}\left(\hat{\theta}_{i}-\theta_{i}\right)^{2} / 3}$ and the power estimation error is defined by er ${ }^{\text {power }} \triangleq$ $\sqrt{\sum_{i=1}^{3}\left(\hat{p}_{i}-p_{i}\right)^{2} / 3}$. Figure 3a,b,d,e represent histograms of $\mathrm{er}_{\mathrm{EIAA}}^{\text {angle }}-\mathrm{er}_{\mathrm{IAA}}^{\text {angle }}$ and $\mathrm{er}_{\mathrm{EIAA}}^{\text {power }}-\mathrm{er}_{\mathrm{IAA}}^{\text {power }}$, and the triangles indicate the centroids of the histograms. It is obvious that most values of er $\mathrm{er}_{\mathrm{EIAA}}^{\text {angle }}-\mathrm{er}_{\text {IAA }}^{\text {angle }}$ and $\mathrm{er}_{\mathrm{EIAA}}^{\text {power }}-\mathrm{er}_{\text {IAA }}^{\text {power }}$ are close to zero, which implies that EIAA is comparable with IAA in terms of the accuracy of angle and power estimation. Moreover, the negative centroids indicate that the estimation accuracy of EIAA is slightly better than that of IAA. Figure $3 \mathrm{c}, \mathrm{f}$ represent the histograms of the running time ratio (RTR) of EIAA and IAA, and the triangles indicate the histogram centroids, and it can be seen EIAA is certainly faster than IAA since all results of RTR are smaller than one. Moreover, the centroids of RTR about 0.1 indicate that the computational efficiency is significantly improved by the proposed EIAA. For various SNR, the performances of IAA and EIAA are compared in Table 3, where each result is obtained by finding the centroid position of the histogram over 500 trials (see triangle position in Figure 3 for example). One can see that EIAA has slightly better angle and power estimation accuracy than IAA. As for the computational efficiency, the running time of EIAA is less than $15 \%$ of that of IAA for various SNR.

\section{Conclusion}

In this article, EIAA algorithm is proposed for source localization. By selecting the principal components of spatial estimate in each iteration, the optimal filter is only utilized to estimate the spatial components likely corresponding to the actual signal sources, and the other spatial components corresponding to noise are updated by the simple correlation of the basis matrix with the residue. Compared with the original IAA that performs optimal filtering on every scanning angle grid in each iteration, EIAA shows higher computational efficiency and slightly better accuracy of angle and power estimation.

\section{Acknowledgements}

This study was supported in part by the National Natural Science Foundation of China under Grant 40901157, and in part by the National Basic Research Program of China (973 Program) under Grant 2010CB731901, in part by the Doctoral Fund of Ministry of Education of China under Grant 200800031050, and in part by Tsinghua National Laboratory for Information Science and Technology (TNList) Cross-discipline Foundation. Xia's work was supported by the National Science Foundation (NSF) under Grant CCF-0964500 and the World Class Univerrsity (WCU) Program, National Research Foundation, Korea.

\section{Competing interests}

The authors declare that they have no competing interests.

\section{Authors' contributions}

$G L$ carried out the algorithm design and the drafted the manuscript. $H Z$ and XW participated in convergence analysis. X-GX participated in statistical analysis. All authors read and approved the final manuscript.

\section{Author details}

${ }^{1}$ Tsinghua National Laboratory for Information Science and Technology (TNList), Department of Electronic Engineering, Tsinghua University, Beijing 100084, China. ${ }^{2}$ Department of Electrical and Computer Engineering, University of Delaware, Newark, DE 19716, USA.

Received: 11 July 2011 Accepted: 12 January 2012

Published: 12 January 2012

\section{References}

1. J Capon, High resolution frequency-wavenumber spectrum analysis. Proc IEEE 57(8), 1408-1418 (1969)

2. RO Schmidt, Multiple emitter location and signal parameter estimation. IEEE Trans Antennas Propag 3, 276-280 (1986)

3. R Roy, T Kailath, ESPRIT - estimation of signal parameters via rotational invariance techniques. IEEE Trans Acoust Speech Signal Process 37(7), 984-995 (1989)

4. S Bourennane, C Fossati, J Marot, About noneigenvector source localization methods. EURASIP J Adv Signal Process 2008 (2008). Article ID 480835

5. Q Wang, Q Jiang, Simulation of matched field processing localization based on empirical mode decomposition and Karhunen-Loeve expansion in underwater waveguide environment. EURASIP J Adv Signal Process 2010 (2010). Article ID 483524

6. IF Gorodnitsky, BD Rao, Sparse signal reconstruction from limited data using FOCUSS: a re-weighted minimum norm algorithm. IEEE Trans Signal Process 45(3), 600-616 (1997)

7. X Tan, W Roberts, J Li, P Stoica, Sparse learning via iterative minimization with application to MIMO radar imaging. IEEE Trans Signal Process 59(3), 1088-1101 (2011)

8. T Yardibi, J Li, P Stoica, M Xue, AB Baggeroer, Source localization and sensing: a nonparametric iterative adaptive approach based on weighted least squares. IEEE Trans Aerosp Electron Syst 46(1), 425-443 (2010)

9. W Roberts, P Stoica, J Li, T Yardibi, FA Sadjadi, Iterative adaptive approaches to MIMO radar imaging. IEEE J Sel Top Signal Process 4(1), 5-20 (2010) 
10. P Stoica, J Li, J Ling, Missing data recovery via a nonparametric iterative adaptive approach. IEEE Signal Process Lett 16(4), 241-244 (2009)

11. NR Butt, A Jakobsson, Coherence spectrum estimation from nonuniformly sampled sequences. IEEE Signal Process Lett 17(4), 339-342 (2010)

12. $M X u e, L X u, J$ Li, IAA spectral estimation: fast implementation using the Gohberg-Semencul factorization. IEEE Trans Signal Process 59(7), 3251-3261 (2011)

13. GO Glentis, A Jakobssony, Efficient implementation of iterative adaptive approach spectral estimation techniques. IEEE Trans Signal Process 59(9), 4154-4167 (2011)

14. J Sun, J Tian, G Wang, S Mao, Doppler ambiguity resolution for multiple PRF radar using iterative adaptive approach. Electron Lett 46(23), 1562-1563 (2010)

15. G Li, J Xu, Y-N Peng, X-G Xia, An efficient implementation of a robust phase unwrapping algorithm. IEEE Signal Process Lett 14(6), 393-396 (2007)

16. W Wang, X-G Xia, A closed-form Robust Chinese Remainder Theorem and its performance analysis. IEEE Trans Signal Process 58(11), 5655-5666 (2010)

doi:10.1186/1687-6180-2012-7

Cite this article as: Li et al.: An efficient implementation of iterative adaptive approach for source localization. EURASIP Journal on Advances in Signal Processing 2012 2012:7.

Submit your manuscript to a SpringerOpen ${ }^{\circ}$ journal and benefit from:

- Convenient online submission

- Rigorous peer review

- Immediate publication on acceptance

- Open access: articles freely available online

- High visibility within the field

- Retaining the copyright to your article

Submit your next manuscript at $\boldsymbol{s p r i n g e r o p e n . c o m ~}$ 